\title{
Apoa5 Q139X truncation predisposes to late-onset hyperchylomicronemia due to lipoprotein lipase impairment
}

\author{
Christophe Marçais,, ${ }^{1,2}$ Bruno Verges, ${ }^{3}$ Sybil Charrière, ${ }^{2,4}$ Valérie Pruneta, ${ }^{2}$ Micheline Merlin, ${ }^{1}$ \\ Stéphane Billon, ${ }^{1}$ Laurence Perrot, ${ }^{4}$ Jocelyne Drai, ${ }^{1}$ Agnès Sassolas, ${ }^{5}$ Len A. Pennacchio, ${ }^{6}$ \\ Jamila Fruchart-Najib, ${ }^{7}$ Jean-Charles Fruchart, ${ }^{7}$ Vincent Durlach, ${ }^{8}$ and Philippe Moulin ${ }^{2,4}$
}

${ }^{1}$ Laboratoire de Biochimie, Centre Hospitalier Lyon-Sud, Hospices Civils de Lyon, Pierre-Benite Cedex, France. ${ }^{2}$ UMR 585 INSERM/INSA de Lyon, Villeurbanne, France. ${ }^{3}$ U498 INSERM, Services d'Endocrinologie, Centre Hospitalier Universitaire (CHU) du Bocage, Dijon, France. ${ }^{4}$ Fédération d'Endocrinologie, Maladie Métaboliques, Diabète et Nutrition, ${ }^{5}$ Laboratoire de Biochimie, Hôpital Louis Pradel, Hospices Civils de Lyon, France. ${ }^{6}$ Department of Genome Sciences, Lawrence Berkeley National Laboratory, Berkeley, California, USA, and the Joint Genome Institute, Walnut Creek, California, USA. ${ }^{7}$ U545 INSERM, Institut Pasteur de Lille and Faculte de Pharmacie de Lille, Lille Cedex, France. ${ }^{8}$ Service d’ Endocrinologie, Maladies Métabolique et Médecine Interne, Hopital Robert Debré, Reims Cedex, France.

\begin{abstract}
While type 1 hyperlipidemia is associated with lipoprotein lipase or apoCII deficiencies, the etiology of type 5 hyperlipidemia remains largely unknown. We explored a new candidate gene, $A P O A 5$, for possible causative mutations in a pedigree of late-onset, vertically transmitted hyperchylomicronemia. A heterozygous Q139X mutation in $A P O A 5$ was present in both the proband and his affected son but was absent in 200 controls. It was subsequently found in 2 of 140 cases of hyperchylomicronemia. Haplotype analysis suggested the new Q139X as a founder mutation. Family studies showed that 5 of 9 total Q139X carriers had hyperchylomicronemia, 1 patient being homozygote. Severe hypertriglyceridemia in 8 heterozygotes was strictly associated with the presence on the second allele of 1 of 2 previously described triglyceride-raising minor APOA5 haplotypes. Furthermore, ultracentrifugation fraction analysis indicated in carriers an altered association of Apoa5 truncated and WT proteins to lipoproteins, whereas in normal plasma, Apoa5 associated with VLDL and HDL/LDL fractions. APOB100 kinetic studies in 3 severely dyslipidemic patients with Q139X revealed a major impairment of VLDL catabolism. Lipoprotein lipase activity and mass were dramatically reduced in dyslipidemic carriers, leading to severe lipolysis defect. Our observations strongly support in humans a role for APOA5 in lipolysis regulation and in familial hyperchylomicronemia.
\end{abstract}

\section{Introduction}

Raised plasma triglyceride (TG) levels are an independent risk factor for coronary artery disease (1) and are influenced by both genetic and environmental factors. Severe hypertriglyceridemia (HTG) is a general condition with a few well-documented genetic contributors, including lipoprotein lipase ( $L P L), A P O C 2$, and $A P O E$, as well as environmental factors such as diet and/or conditions such as pregnancy and diabetes $(2-5)$. While genetic factors account for a large proportion of the rare type 1 hyperlipidemia, the complex interaction between genetics and environment is only partly understood in the more common type 5 hyperlipidemia.

A strong candidate for severe HTG is the recently discovered human apolipoprotein $A-V(A P O A 5)$ gene based on its profound modulation of plasma TG concentration. In mice, apoa5 overexpression lowered plasma TG concentration (6-8) whereas mice lacking Apoa5 had a 4-fold increase in plasma TG concentration (6). In humans, independent studies have demonstrated that variant haplotypes with either the S19W or the c.A-3G APOA5 polymorphisms are strong determinants of plasma TG

Nonstandard abbreviations used: APOA5, human apolipoprotein A-V; Apoa5, murine apolipoprotein A-V; FCR, fractional catabolic rate; HSPG, heparan sulfateproteoglycan; HTG, hypertriglyceridemia; LPL, lipoprotein lipase; PR, production rate; SNP, single nucleotide polymorphism; TC, total cholesterol; TG, triglyceride. Conflict of interest: The authors have declared that no conflict of interest exists. concentration variability across human populations (9-11). To address whether APOA5 is a genetic contributor to TG metabolism and disease, we sequenced this candidate gene in a pedigree of vertically transmitted hyperchylomicronemia without LPL or apoCII deficiencies.

\section{Results}

\section{APOA5-Q139X gene mutation in byperchylomicronemia}

The APOA5 gene was studied in family A (Figure 1) because of its unusual features, late onset of hyperchylomicronemia with vertical transmission and resistance to diet and lipid-lowering treatments, whereas $L P L$ and $A P O C 2$ deficiencies were excluded. Sequence analysis of APOA5 in the proband from family A (AII1) revealed he was heterozygous for a unique C1047T transition at the first nt of codon 139 (CAG) in exon 3 generating a Q139X nonsense mutation (Figure 1).

The presence of the Q139X allele was subsequently studied in a group of 200 unrelated control subjects who all scored negative (allele frequency $<0.25 \%$ ) and in 140 patients with a history of documented HTG (TG > $15 \mathrm{mM} / \mathrm{l}$ ), 2 of which were found to be positive. One heterozygous patient (BIII3) led to the identification of the second larger family B (Figure 1) and the other (CI1) was homozygous, but his death impeded further phenotype studies. Haplotype study following subcloning and sequencing of large $7-\mathrm{kb}$ PCR products indicated in the 2 probands (AII1, 


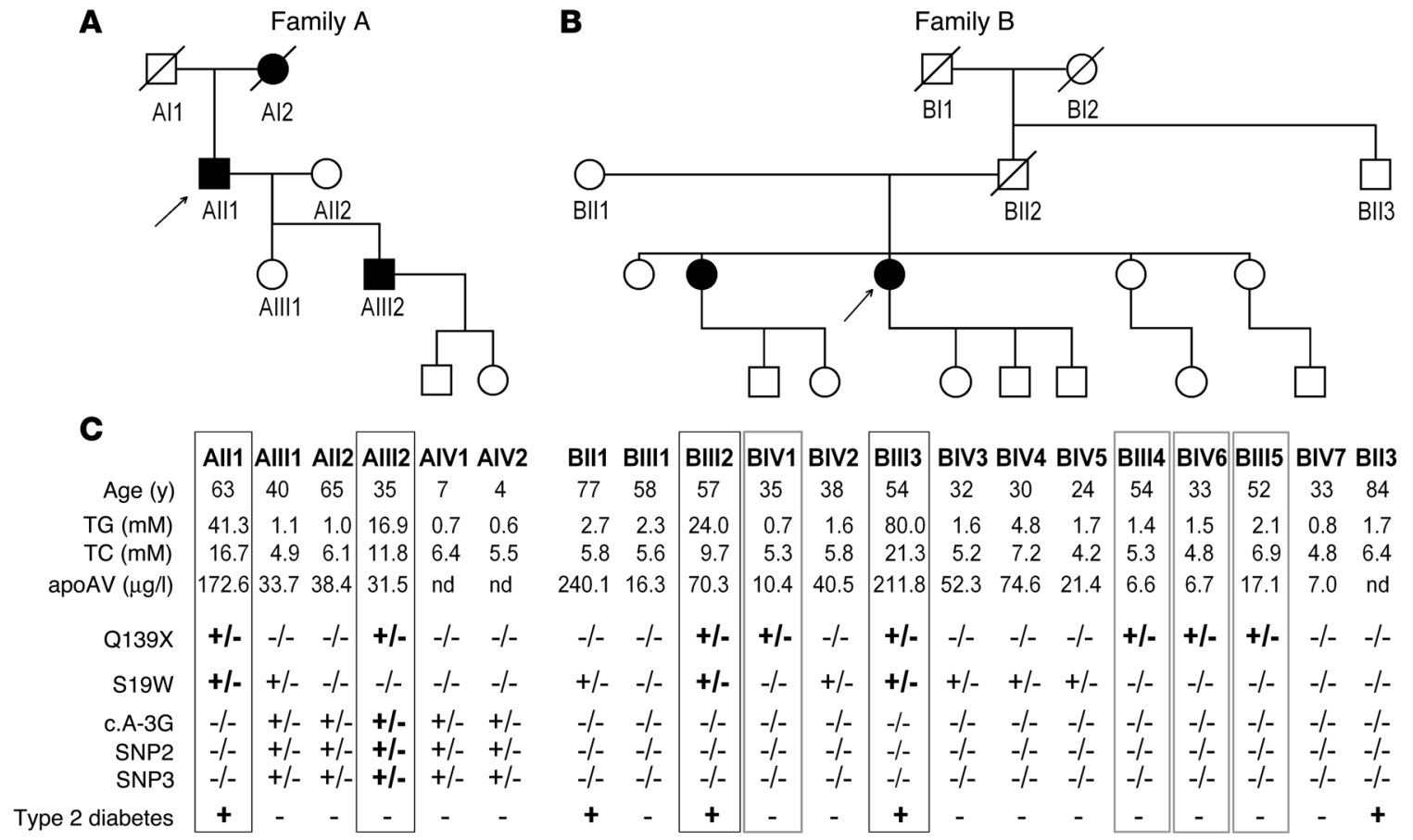

\section{Figure 1}

Pedigree charts of family A (A) and family B (B). Arrows, probands; filled circles or filled squares, patients with confirmed severe type 5 hyperlipoproteinemia. (C) Results for the Q139X carriers are indicated in boxes. Black boxes, patients with severe chylomicronemia; gray boxes, carriers without severe chylomicronemia; -/-, homozygote genotype for the major allele; +/-, heterozygote genotype; +/+, homozygote genotype for the minor allele; ND, not determined. SNP and mutations are detailed in Table 1.

BIII3) and the homozygote (CI1) that all 3 patient had an identical Q139X-bearing haplotype from $n t-2700$ to +2000 . This haplotype also had the minor g.A-2200T and g.C-1464T alleles, suggesting that Q139X is a founder mutation (Table 1). In the heterozygote patients AII 1 and BIII3, the second APOA5 allele was shown to harbor only previously reported polymorphisms, specifically the S19W variant which was absent from the Q139Xbearing haplotype (Table 1).

\section{Case reports}

Family A. The index case (AII1), a 63-year-old white male, was referred for a severe unmanageable HTG. His first episode (TG: $22 \mathrm{mM} / \mathrm{l}$ ) was discovered when he was 38 . His lipid profile was initially normalized by dietary restriction, but he relapsed in his $40 \mathrm{~s}$ and 50s despite optimal dietary adherence. Severe HTG became permanent when he reached 60, fluctuating between 15 and 40 $\mathrm{mM} / 1 \mathrm{TG}$ with transiently higher concentrations of over $60 \mathrm{mM} / 1$. He never suffered from acute pancreatitis and showed no evidence of coronary heart disease (negative maximal treadmill test); however, his carotid intima media thickness was increased $(0.90$ $\mathrm{mm}$ ), and he had atheromatous plaques both in the carotid and the aorta. He had no cause of secondary dyslipidemia except for a mild type 2 diabetes diagnosed when he was $63(\mathrm{HbA} 1 \mathrm{c}=6.5 \%$; $\mathrm{BMI}=26$; waist circumference $=96 \mathrm{~cm}$ ). Fibrates and high doses of long-chain omega-3 FFAs were unsuccessfully tested. His mother, who died at 94, had a history of plasma TG concentrations reaching $18 \mathrm{mM} / \mathrm{l}$ (AI2). His 34-year-old son of normal wt (AIII2) was found severely hypertriglyceridemic at 29 ( TG $=28 \mathrm{mM} / \mathrm{l}$; total cholesterol $[\mathrm{TC}]=8.4 \mathrm{mM} / \mathrm{l}$ ) although at 24 , his fasting plasma
TG levels had been found normal $(1.46 \mathrm{mM} / \mathrm{l})$. Under dietary advice and intensive exercise training, he was able to normalize his plasma TG concentrations but sometimes reached 8.3 and 11 $\mathrm{mM} / \mathrm{l}$. However, he has become permanently hypertriglyceridemic for the past 4 years (TG $=10 \mathrm{mM} / \mathrm{l} ; \mathrm{TC}=6 \mathrm{mM} / \mathrm{l}$ ) although he has no identified cause of secondary dyslipidemia.

PAGE of plasma lipoproteins from both cases in family A showed a type 5 hyperlipoproteinemia with accumulation of chylomicrons and VLDL with low LDL and HDL. Plasma apoCII and apoCIII concentrations were found elevated with a mild increase in the apoCII/apoCIII ratio. APOE genotype was determined to be E2E3 in both AII1 and AIII2. Direct sequencing of the LPL gene in both patients did not reveal deleterious mutations. Overall, the presentation was a severe HTG with hyperchylomicronemia, resistant to treatment over at least 3 generations.

Family B. A second, unrelated larger family with a similar phenotype was subsequently discovered (Figure $1 \mathrm{~B}$ ). The index case was a 54-year-old white female (BIII3) exhibiting severe HTG $(\mathrm{TG}=68 \mathrm{mM} / \mathrm{l} ; \mathrm{TC}=30.3 \mathrm{mM} / \mathrm{l})$. She had a history of hypertension, glucose intolerance, and moderate HTG (TG $<8.5 \mathrm{mM} / \mathrm{l})$ for 13 years with abdominal obesity $(\mathrm{BMI}=34$; waist circumference $=102 \mathrm{~cm}$ ). Subsequently, she has presented type 2 diabetes efficiently treated by metformine $(\mathrm{HbA} 1 \mathrm{c}=6.7 \%)$. She had no additional secondary cause of HTG. It took more than 2 weeks to lower her plasma TG levels to less than $10 \mathrm{mM} / 1$ despite intensive dietary intervention with transient insulinotherapy followed by a combination of metformine $(1 \mathrm{~g} / \mathrm{d})$, fenofibrate $(300 \mathrm{mg} / \mathrm{d})$, and pioglitazone $(15 \mathrm{mg} / \mathrm{d})$. Five months after, she exhibited another acute episode of type 5 lipidemia $(\mathrm{TG}=80$ 
Table 1

APOA5 haplotypes in patient with severe hyperchylomicronemia

\begin{tabular}{|c|c|c|c|c|c|}
\hline SNP/mutation & dbSNP identifier ${ }^{A}$ & Apoa5 & Haplotype I & $\begin{array}{c}\text { Haplotype II } \\
\mathrm{APOA}^{*} 2^{\mathrm{B}}\end{array}$ & $\begin{array}{c}\text { Haplotype III } \\
\mathrm{APOA}^{*} 3^{\mathrm{B}}\end{array}$ \\
\hline g.A-2200T & rs1787680 & & $\mathrm{T}$ & A & A \\
\hline g.C-1464T & SNP in All1 genome ${ }^{C}$ & & $\mathrm{~T}$ & C & C \\
\hline g.T-1131C (SNP3) & rs662799 & & $\mathrm{T}$ & C & $\mathrm{T}$ \\
\hline g.A-3G (Kozak) & rs651821 & & $A$ & G & A \\
\hline g.C170G & rs3135506 & S19W & C & C & G \\
\hline g.C245A & rs12287036 & $|44|$ & C & C & $A$ \\
\hline g.G751A (SNP2) & rs2072560 & & G & $A$ & $\mathrm{G}$ \\
\hline g.c1047T & $\begin{array}{c}\text { Mutation in } \\
\text { All1 genome }\end{array}$ & Q139X & $\mathrm{T}$ & C & C \\
\hline \multicolumn{6}{|l|}{ Patients } \\
\hline & All1 & & + & - & + \\
\hline & Alll2 & & + & + & - \\
\hline & BIII2 & & + & - & + \\
\hline & BIII3 & & + & - & + \\
\hline & $\mathrm{Cl} 1$ & & + & - & - \\
\hline
\end{tabular}

${ }^{A}$ As shown on the Single Nucleotide Polymorphism database (http://www.ncbi.nlm.nih.gov/projects/ SNP/). ${ }^{B}$ APOA5 haplotype as defined in ref. 9 on the basis of genotypes at SNP1, SNP2, and SNP3. CPreviously unreported entries, which have not yet been assigned dbSNP identifiers.
He was not diabetic, of normal wt $(70 \mathrm{~kg}$; for $1.71 \mathrm{~m}$ height; waist circumference $=90$ $\mathrm{cm}$ ) and only presented transient fasting hyperglycemia upon acute peak of HTG. Except for beta blocker introduced after myocardial infarction, he had no additional cause of secondary dyslipidemia. His brother had a mild mixed hyperlipidemia and his sister was normolipidemic. Overall, the presentation was a late onset fasting hyperchylomicronemia over a period of 30 years, resistant to treatment.

\section{APOA5-Q139X and haplotype study in pedigrees}

Members of both families were screened for the presence of the Q139X allele; 6 additional heterozygote carriers were identified, 1 from family A and 5 from family B (Figure 1). Fasting hyperchylomicronemia had occurred in 5 out of 9 carriers (AII1, AIII2, BIII2, BIII3, and CI1) but not in any of the 10 adult noncarriers tested from both families (Figure 1, A and B).
$\mathrm{mM} / \mathrm{l})$ with similar evolution despite wt stability and an efficient glycemic control ( $\mathrm{HbA} 1 \mathrm{c}=6.5 \%)$.

Family history was associated with type 2 diabetes on the maternal side, but the exact severity of HTG in subjects BI1 and BII2 was unknown. Additionally, all the proband's 4 sisters had abdominal obese phenotypes (BIII1, 2, 4, 5); 2 had mild (BIII1 and BIII5) and 1 severe (BIII2) HTG. BIII2, a 56-year-old female, displayed HTG ranging between 7 and $10 \mathrm{mM} / 1$ under various unsuccessful treatments (fibrates, long-chain omega-3 fatty acid). Her highest recorded triglyceridemia was $24 \mathrm{mM} / 1$ without history of pancreatitis. She has been obese $(\mathrm{BMI}=40.1$; waist circumference $=113$ $\mathrm{cm}$ ) and hypertensive for more than 30 years and had recently been diagnosed with type 2 diabetes $(\mathrm{HbA} 1 \mathrm{c}=7.1 \%)$. PAGE of plasma lipoproteins from BIII2 and BIII3 revealed type 5 hyperlipoproteinemia. Their APOE genotypes were respectively E3E3 and E3E4.

As in family A, the overall presentation was a familial severe hyperchylomicronemia highly resistant to treatment with WT LPL genes and elevated plasma apoCII concentrations. Additionally, in family B this dyslipemia was closely associated with overt abdominal obesity and mild type 2 diabetes.

Patient CI1. Hyperchylomicronemia was discovered at 34 in this white male upon the occurrence of acute pancreatitis. He displayed a permanent unmanageable HTG during 20-year follow-up with 3 additional episodes of acute pancreatitis. At 49, he presented a silent myocardial infraction. At 60 , he died from a cause unrelated to his dyslipidemia.

Most of the time, his plasma TG concentrations ranged between 15 and $30 \mathrm{M} / 1$ with several peaks above $40 \mathrm{mM} / 1$ (maximal range 6.5-65 mM/l) despite strict dietary compliance and a combination of fibrate and omega- 3 fatty acids. He had type 5 hyperlipidemia, as determined by electrophoresis of plasma lipoprotein and ultracentrifugation, with normal plasma apoB concentration $(1.1 \mathrm{~g} / \mathrm{l})$, low LDLc and HDLc (0.58 and $1.1 \mathrm{mM} / 1$, respectively), and 3 times more TGs in chylomicrons than in VLDL fraction. APOE genotype was $E 3 / E 3$; the presence of $L P L$ gene mutation was excluded, and plasma apoCII/apoCIII ratio was normal.
All 5 Q139X carriers with severe HTG were either homozygote (CI1) or heterozygote with minor haplotypes on the second APOA5 allele (Table 1). In family A (Figure 1A), the proband (AII1) transmitted Q139X to his son (AIII2). AII1 also had the S19W signal peptide variant (APOA5*3 haplotype) whereas his son (AIII2) had the maternal $c . A-3 G$ allele with the minor alleles for single nucleotide polymorphism 2 (SNP2) and SNP3 (APOA5*2 haplotype). In family $\mathrm{B}$ (Figure 1B), the 2 severely dyslipidemic Q139X carriers (proband BIII3 and BIII2) had the S19W polymorphism on the second allele (APOA5*3 haplotype). In contrast, the 4 carriers identified without history of severe HTG had the most common $A P O A 5^{*} 1$ haplotype on the second chromosome. Although in their fifties, BIII4 remained normolipidemic, and BIII5 had only moderate HTG. The 2 normolipidemic carriers from the younger generation, BIV1 and BIV6, are only in their thirties.

\section{Apoa5 distribution in plasma lipoproteins}

In both families, murine apolipoprotein A-V (Apoa5) plasma concentrations were not related to the presence of Q139X (Figure 1). Q139X is predicted to determine a truncation of Apoa5 at residue 116 of the mature protein, generating a $15-\mathrm{kDa}$ peptide. In order to assess the presence of the putative truncated peptide in the plasma of heterozygotes, we analyzed by Western blotting the Apoa5 distribution in lipoprotein subfractions obtained following ultracentrifugation. A $15-\mathrm{kDa}$ peptide containing the $\mathrm{N}$ terminal epitopes of Apoa5 was found in the 6 Q139X heterozygotes tested but not in 3 noncarriers or 10 unrelated normolipidemic controls (Figure 2 ). This $15-\mathrm{kDa}$ peptide was exclusively located in the unbound fraction $(d>1.21)$. The $40-\mathrm{kDa}$ full-length Apoa5 was detected in plasma-derived VLDL and HDL fractions from the noncarriers and unrelated normolipidemic controls. In contrast, in the 6 Q139X heterozygotes tested, both the $40-\mathrm{kDa}$ and the $15-\mathrm{kDa}$ truncated Apoa5 were recovered exclusively from the unbound fraction (Figure 2). As shown in Figure 2, this altered association of WT Apoa5 to lipoproteins was present in all the carriers whether they were hyperlipidemic (AII1, AIII2, BIII2, and BIII3) or not (BIII4 and BIII5). 

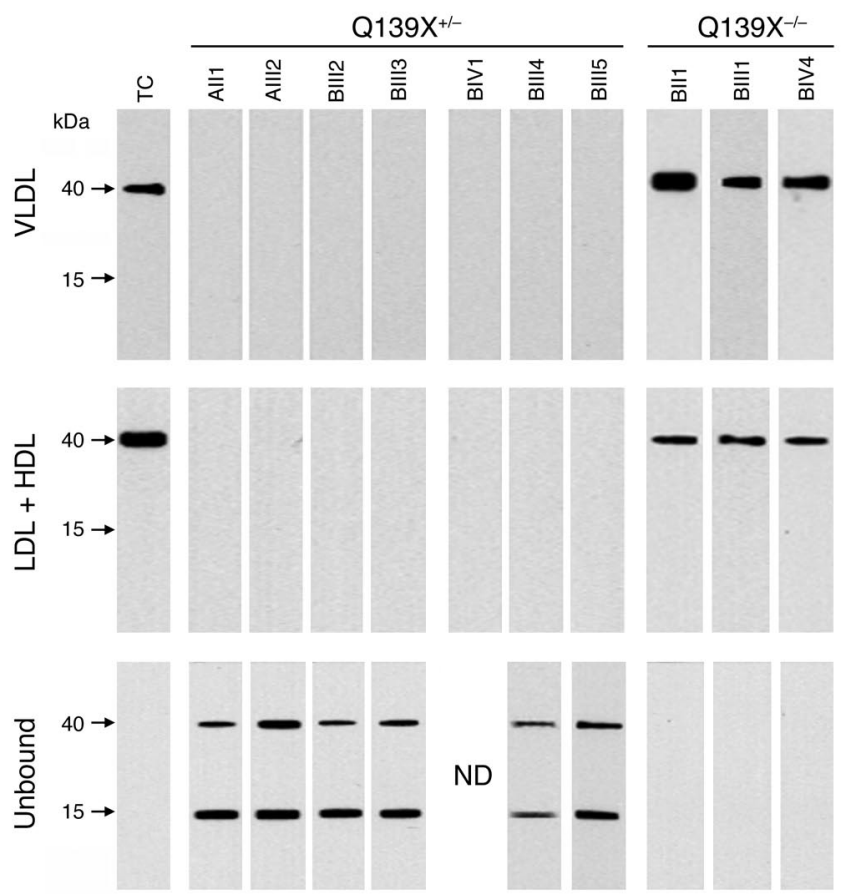

Figure 2

Western blot analysis of Apoa5 distribution in plasma lipoproteins. Ultracentrifugation fractions were as follows: VLDL fraction, $d<1.006$ $\mathrm{g} / \mathrm{ml}$; LDL + HDL, $1.019<d<1.210$; unbound to lipoproteins, $d>1.21$. TC is representative of the analysis of plasma lipoprotein fraction of 10 unrelated normolipidemic controls.

\section{Plasma APOB100 kinetics}

Plasma APOB100 kinetics were studied in 3 patients with Q139X mutation (AII1, AIII2 and BIII3). Kinetic curves (Figure 3) clearly show a much slower isotopic enrichment of VLDL-apoB compared with the normolipidemic control subjects, indicating a decreased VLDL-apoB catabolic rate. As shown in Table 2, severely dyslipidemic patients with Q139X had a 20- to 44-fold increase of VLDL-apoB pool due to a 22- to 26-fold decrease in VLDL-apoB fractional catabolic rate (FCR) (transfer to IDL/LDL). VLDL-apoB production rates (PRs) were identical to controls in 2 patients (AII1 and AIII2) and only slightly increased in 1 patient (BII3).

The 3 patients had a significant increase of IDL-apoB due to a 2.6- to 15 -fold reduction of apoB-IDL FCR. This reduction was entirely attributable to the low apoB-IDL indirect FCR (transfer to LDL) whereas apoB-IDL direct FCR was not reduced in the patients (Table 2). As a consequence, LDL-apoB PRs were reduced in the 3 patients with conserved LDL-apoB FCRs, resulting in a decrease of LDL-apoB pool (Table 2). These results indicate a major alteration in the lipolysis of TG-rich lipoproteins in 3 heterozygote Q139X carriers.

\section{LPL defect}

To further assess the lipolysis defect associated with dyslipidemia in Q139X carriers, LPL activity was measured in postheparin plasma from all the severely dyslipidemic patients with Q139X (CI1, AII1, AIII2, BIII2, and BIII3) and from 2 carriers without severe HTG (BIII4 and BIII5). Postheparin LPL activities were dramatically reduced in all 5 severely hyperlipidemic Q139X carriers as compared with unrelated controls (Figure 4). The residual activities were similar to those found in homozygous $L P L$ deficiency. By contrast, BIII4 and BIII5 had normal LPL activities (Figure 4). Hepatic lipase activity in postheparin plasma from 4 severely dyslipidemic patients (AII1, AIII2, BIII2, and BIII3) was similar to that found in controls ( $7.6 \pm 2.7$ vs. $7.7 \pm 2.3 \mu \mathrm{M} \mathrm{FFA} / \mathrm{ml} / \mathrm{h}, P=$ not significant). We subsequently analyzed LPL protein in postheparin plasma by Western blotting to determine whether functionally inactive LPL was released or LPL was not released. LPL protein was present in postheparin plasma from 2 normolipidemic Q139X carriers (BIII4 and BIII5) as in the controls but remained undetectable in the postheparin plasma from the 5 severely hypertriglyceridemic carriers: AII1, AIII2, BIII2, BIII3, and CI1 (Figure 4).

\section{Discussion}

We report what we believe to be a new form of familial hyperchylomicronemia with vertical transmission, late onset, incomplete penetrance, and an unusual resistance to conventional treatment. Our results strongly suggest that the newly identified APOA5-Q139X private nonsense mutation is a determinant of this new dyslipidemia based on the following: (a) observation in the 2 pedigrees that hyperchylomicronemia occurs only in the Q139X carriers and cosegregates with the Q139X mutant allele in pedigree A; (b) presence in the carriers of a $15-\mathrm{kDa}$ peptide corresponding to the $\mathrm{N}$ terminal end of Apoa5, as expected for a truncation at residue 139; and (c) altered association of WT Apoa5 to plasma lipoproteins in all the carriers from both families. Additionally (d), we demonstrate that severe HTG in Q139X mutation carriers results from an LPL defect leading to lipolysis impairment.

In family $\mathrm{A}$, the fasting hyperchylomicronemia was expressed in at least 3 consecutive generations. Moreover, in both pedigrees this dyslipidemia occurred exclusively among the Q139X carriers, none of which had an identifiable deleterious mutation of the second APOA5 allele. This, together with similar dyslipidemia in the homozygote CI1 and 3 heterozygote carriers (AII1, AIII2, and BIII3), initially suggested that Q139X caused a dominant hyperchylomicronemia. But subsequent study of family B indicated the Q139X $X^{+/-}$genotype was incompletely penetrant with only 2 out of 6 carriers affected in family B. A role for compound heterozygocity with either the $A P O A 5 * 3$ (with S19W) or the APOA5*2 haplotype (with c.A-3G, SNP2, and SNP3 minor alleles) (Table 1) was indicated by the complete association of hyperchylomicronemia with these compound heterozygote genotypes. Two normolipidemic carriers

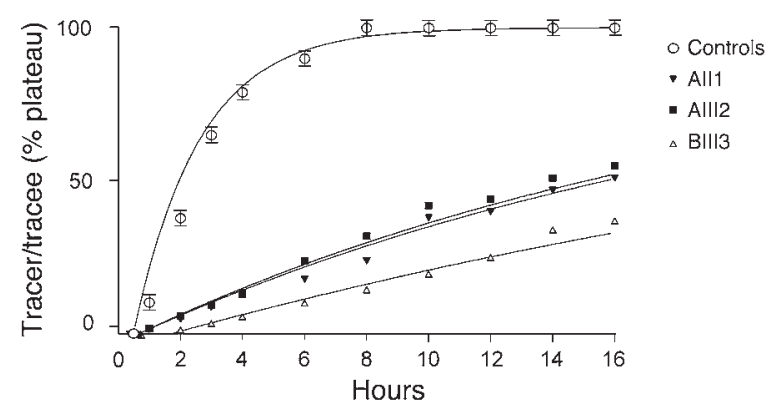

Figure 3

Kinetic curves of VLDL-apoB in plasma. ${ }^{13} \mathrm{C}$-leucine enrichment curves of human APOB100 in patients All1 (filled triangle), Alll2 (filled squares), and BIII3 (open triangles) and in 10 controls (open circles). Results are expressed as tracer/tracee values (mean of triplicates). For controls, data are shown as mean \pm SEM. 


\section{Table 2}

Kinetic parameters of VLDL, IDL, and LDL apoB in 3 Q139X mutated patients and in control subjects

\begin{tabular}{|c|c|c|c|c|c|c|c|c|c|c|c|}
\hline & \multicolumn{4}{|c|}{ VLDL apoB } & \multicolumn{4}{|c|}{ IDL apoB } & \multicolumn{3}{|c|}{ LDL apoB } \\
\hline & \multirow[t]{2}{*}{$\begin{array}{c}\text { apoB } \\
(\mathrm{mg} / \mathrm{l})\end{array}$} & \multirow[t]{2}{*}{$\begin{array}{l}\text { Production } \\
(\mathrm{mg} / \mathrm{kg} / \mathrm{d})\end{array}$} & \multicolumn{2}{|c|}{$\begin{array}{c}\text { FCR } \\
\text { (pool/d) }\end{array}$} & \multirow[t]{2}{*}{$\begin{array}{l}\text { apoB } \\
(\mathrm{mg} / \mathrm{l})\end{array}$} & \multirow[t]{2}{*}{$\begin{array}{l}\text { Production } \\
(\mathrm{mg} / \mathrm{kg} / \mathrm{d})\end{array}$} & \multicolumn{2}{|c|}{$\begin{array}{c}\text { FCR } \\
\text { (pool/d) }\end{array}$} & \multirow[t]{2}{*}{$\begin{array}{l}\text { apoB } \\
(\mathrm{mg} / \mathrm{l})\end{array}$} & \multirow[t]{2}{*}{$\begin{array}{l}\text { Production } \\
(\mathrm{mg} / \mathrm{kg} / \mathrm{d})\end{array}$} & \multirow[t]{2}{*}{$\begin{array}{c}\text { FCR } \\
\text { (pool/d) }\end{array}$} \\
\hline & & & $\begin{array}{l}\text { Direct } \\
\text { catab. }\end{array}$ & $\begin{array}{l}\text { Transfer } \\
\text { to IDL/LDL }\end{array}$ & & & $\begin{array}{l}\text { Direct } \\
\text { catab. }\end{array}$ & $\begin{array}{l}\text { Transfer } \\
\text { to LDL }\end{array}$ & & & \\
\hline Patient All1 & 743 & 17.0 & 0 & 0.51 & 112 & 17.1 & 2.1 & 1.28 & 164 & 6.6 & 0.90 \\
\hline Patient AIII2 & 658 & 14.2 & 0 & 0.48 & 197 & 14.0 & 0.6 & 0.98 & 239 & 8.9 & 0.83 \\
\hline $\begin{array}{l}\text { Control men }{ }^{A} \\
(n=5)\end{array}$ & $33 \pm 4$ & $14.8 \pm 4.1$ & 0 & $11.3 \pm 4.0$ & $39 \pm 9$ & $17.5 \pm 7.0$ & $1.4 \pm 0.9$ & $7.4 \pm 4.1$ & $771 \pm 180$ & $26.9 \pm 8.2$ & $0.82 \pm 0.24$ \\
\hline Patient BIII3 & 1562 & 30.2 & 0 & 0.43 & 597 & 33.8 & 0.98 & 0.28 & 407 & 8.2 & 0.45 \\
\hline $\begin{array}{l}\text { Control women }{ }^{\mathrm{A}} \\
(n=5)\end{array}$ & $35 \pm 3$ & $15.6 \pm 2.3$ & $1.6 \pm 1.8$ & $9.4 \pm 3.3$ & $18.0 \pm 2.0$ & $15.6 \pm 4.6$ & $2.1 \pm 1.6$ & $17.6 \pm 6.3$ & $608 \pm 66$ & $12.0 \pm 4.3$ & $0.47 \pm 0.15$ \\
\hline
\end{tabular}

AValues in mean \pm SD. Catab.; catabolism.

are only in their thirties (BIV1 and BIV6), and we cannot exclude the possibility that they will later display severe HTG. However, 2 carriers are in their fifties and were still either normolipidemic (BIII4) or showed moderate HTG, likely due to abdominal obese phenotype (BIII5). Moreover, a unique other APOA5 deleterious mutation, $Q 148 X$, was reported recently in a pedigree with severe hyperchylomicronemia in a homozygote patient (12). None of the reported 10 heterozygotes had severe HTG, and they all had the frequent $A P O A 5 * 1$ haplotype on the second chromosome (12). Therefore, as in our study, heterozygote nonsense APOA5 mutation alone did not result in severe chylomicronemia. This is consistent with our observation that the 2 variant haplotypes were crucial determinants of dyslipidemia in heterozygote Q139X carriers.

The effect of $A P O A 5 * 2$ and $A P O A 5 * 3$ minor haplotypes on Apoa5 function is unknown. Previous studies demonstrated that $A P O A 5^{*} 2$ and $A P O A 5^{*} 3$ are both strong, independent determinants of plasma TG concentration variability across human populations (6, 9-11, 13-16). Furthermore, several studies suggested that S19W and/or c.A-3G enhances HTG in various physiological and pathological conditions such as pregnancy (17), familial combined hyperlipidemia $(16,18,19)$, and mixed hyperlipidemia (20). Moreover, the S19W variant was a crucial determinant of HTG in APOE2 homozygotes (21). Our results highly strengthen these studies and further highlight the TG-raising properties of the 2 APOA5 minor haplotypes. On the other hand, population studies indicate that homozygocity for these minor haplotypes only results in a mild increase in plasma TG levels $(9,16)$, suggesting only a mild Apoa5 impairment. This may indicate a complex association of genetic and environmental factors in line with the observed late onset of dislipidemia in this study.

We show that severe chylomicronemia in homozygote and heterozygote Q139X carriers was caused by a profound LPL defect, and we provide what we believe to be the first clear evidence in humans of a functional interplay between Apoa5 and LPL. The apoB kinetic data in 3 dyslipidemic APOA5 mutated patients clearly indicate a major alteration in the lipolysis of TG-rich lipoproteins with a striking reduction of VLDL and IDL catabolism. Unfortunately, kinetic study could not be performed in the homozygote CI1 due to his death. The reduction of catabolism was of greater magnitude for VLDL than for IDL, in line with the LPL defect observed in the 5 severely hyperlipemic carriers. A slight increase of VLDL-apoB production likely contributes to the hyperlipidemia in 1 patient (BIII3). It is most likely the consequence of her diabetes mellitus rather than of Apoa5 truncation because her VLDL-apoB PR is as usually observed in type 2 diabetic patients $(22,23)$. Accordingly, recent experimental studies indicated that Apoa5 does not modulate hepatic VLDL production (24) but induces an LPL-dependent acceleration of the catabolism of TG-rich lipoproteins (24-26). The increase in IDL pool despite the reduced VLDL catabolism is likely due to the 22 -fold increase in VLDL pool leading to a residual conversion into LDL.

The defective VLDL catabolism resulted from strikingly decreased LPL activity in all severely dyslipidemic Q139X carriers. Moreover, Q139X homozygocity (CI1) was associated with the lowest postheparin LPL activity (Figure 4), similar to those in homozygotes for nonsense LPL mutations, such as R192X, IVS1$1 G \rightarrow A$, or $Y 288 X$ (data not shown). Q139X nonsense mutation is predicted to encode a truncated Apoa5 variant, missing the entire hydrophobic region with the lipid-binding domain (27) and a putative heparin-binding domain between aa 186 and 227 (28). In our study, the Q139X truncated peptide was poorly bound to VLDL unlike Apoa5 in control subjects. Furthermore, in heterozygote carriers, the binding of Apoa5 expressed from the second allele to lipoproteins was also impaired. This might be critical to Apoa5 dysfunction because recent experimental studies suggested that upregulation of lipolysis by Apoa5 involves its binding both to lipid and to heparan sulfate-proteoglycan (HSPG). It was proposed that Apoa5 accelerates lipolysis by facilitating the interaction of TG-rich lipoproteins to HSPG-bound LPL (24). First, experimental data suggest that Apoa5 upregulates lipolysis by LPL-HSPG complexes but not by free LPL (24). Second, a recent study indicates that Apoa5 binds to HSPG and enhances the binding of VLDL and

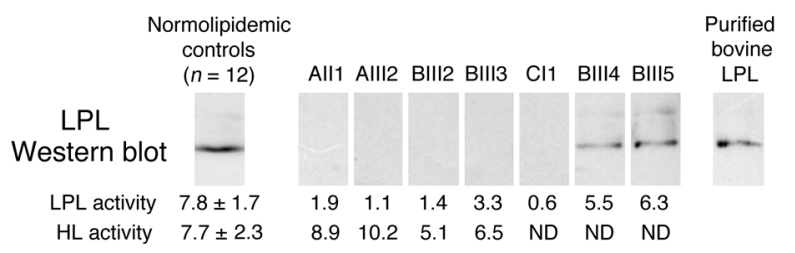

\section{Figure 4}

$\mathrm{LPL}$ activity ( $\mu \mathrm{mol} \mathrm{FFA} / \mathrm{ml} / \mathrm{h}$ ) and mass in postheparin plasma. LPL activity was as follows in patients with LPL deficiency: $2.3 \pm 0.8 \mu \mathrm{mol}$ $\mathrm{FFA} / \mathrm{ml} / \mathrm{h}$ in homozygote LPL deficiency $(n=6)$ and $3.6 \pm 0.7$ in heterozygote $L P L$ deficiency $(n=8)$. HL, hepatic lipase. 
chylomicrons to LPL-HSPG complexes (28). This feature is likely to be defective in the Q139X truncated peptide, resulting in an inability to upregulate lipolysis.

However, LPL protein expression was dramatically decreased on HSPG only in severely dyslipidemic carriers. In contrast, their hepatic lipase activity is normally released by heparin. Hence, rather than simply a defective stimulation of normally expressed LPL, heterozygote carriers who fail to express and/or target LPL to HSPG developed severe HTG whereas those who maintained a normal releasable pool of LPL did not. It remains to be established how LPL expression was altered in the heterozygous carriers as they became hyperchylomicronemic. One possibility is a dominant-negative effect of the $15-\mathrm{kDa}$ truncated Apoa5 expressed in the plasma of all carriers. We speculate that the truncated product could interfere with the normal association of Apoa5 to lipoproteins and affect either indirectly or directly the LPL expression on the endothelium. Such a dominant-negative effect would also account for the severity of lipolysis defect in compound heterozygotes with only mild impairment of the second allele.

The full expression of the syndrome, as observed in the Q139X homozygote CI1 and in all affected heterozygotes, is characterized by type 5 hyperlipidemia, in the thirties, switching from intermittent to permanent severe HTG, unusually resistant to dietary and hypolipidemic drug therapy. Previously, chylomicronemia was diagnosed at 9 years old in the only reported homozygote for a deleterious APOA5 mutation (Q148X) (12). Although we cannot exclude early onset in the Q139X homozygote (CI1), late onset in our study was suggested by late diagnosis in all patients; patient AIII2 was normolipidemic at 24. This suggests in our pedigrees the crucial involvement of environmental factors to modulate the expressivity and timing of dyslipidemia Therefore, this raises the question of how LPL expression was progressively impaired, leading to severe HTG. Studies in apoa5 $\mathrm{KO}$ mice suggested that LPL overexpression can compensate for apoa5 defect and normalize plasma TG concentration (24). Accordingly, we propose that, in our patients, LPL upregulation might have initially compensated for APOA5 deficiency. Later on, age-related factors could have limited LPL availability, which became unable to uphold lipolysis. In family B, there was some association between obesity and severe HTG, but this was not the case in family A, in which wt was normal for both patients. The absence of dietary errors in family A and the lack of response to dietary intervention in all patients suggest that nutritional factors may not be a major determinant of the disease. Type 2 diabetes was present in 3 of 5 patients and has likely favored severe HTG through hepatic VLDL-TG overproduction, as in patient BII3 (Table 2) (23). Furthermore, type 2 diabetes is often associated with mildly reduced LPL activity and mass in fasting and postprandial state (29-31) This could have further impaired lipolysis in addition to Apoa5 deficiency itself. Even though type 2 diabetes has likely contributed to severe HTG in 3 patients (AII1, BIII2, and BIII3), this is not the case for patient AIII2, who remained normoglycemic and normoinsulinemic.

We demonstrate that homozygote APOA5 deficiency due to Q139X nonsense mutation causes familial chylomicronemia through severe impairment of LPL expression. Furthermore, severe heterozygote phenotype can occur, depending on the complex interplay between the rare heterozygous truncation, common susceptibility APOA5 haplotypes, and age-related factors. Overall, our results strongly support a role for the APOA5 gene in the regulation of the lipolysis of TG-rich lipoproteins in humans.

\section{Methods}

\section{Patient assessment}

Probands and pedigrees are detailed in Results. A total of 140 unrelated patients with hyperchylomicronemia were selected when referred to the Hôpital Louis Pradel lipid clinics on the basis of current or history of documented transient episodes of severe fasting HTG. The presence of hyperchylomicronemia was assumed when plasma TG concentration was above $15 \mathrm{mM} / 1$ with a TG/TC ratio above 2.5 (in g/l) (32). The study was performed according to the requirements and approval of the Comité Consultatif d'Éthique (Dijon, France), and written informed consent was obtained from all the patients included in the study.

\section{APOA5 genomic sequence analysis}

APOA5 gene mutation analysis. Genomic DNA was extracted as described (5). Prior to direct sequencing of the APOA5 (nt -25 to +1820 ), genomic DNA $(0.2 \mu \mathrm{g})$ was subjected to PCR with the 2 primers APOA5gF1 (5'-CAGGTGGGCAGGGGAGAGGTGGTA-3') and APOA5gR1 (5'-ATGGCAGCCCTGGGGAGACAAGTG-3') generating a 2526-bp product. PCR was performed with Taq-polymerase $(40 \mathrm{mU} / \mu \mathrm{l})$ as indicated (Qbiogene Inc.), primers $(0.4$ $\mu \mathrm{M} / \mathrm{l}$ each), DMSO $8 \%$ (Sigma-Aldrich), $\mathrm{MgCl} 2$ (4.5 mM/l), and dNTP (0.2 $\mu \mathrm{m} / \mathrm{l})$. A total of 35 cycles were performed at a denaturating temperature of $95^{\circ} \mathrm{C}$ for 40 seconds followed by annealing temperature of $68^{\circ} \mathrm{C}(1 \mathrm{~min})$ and extension temperature of $72^{\circ} \mathrm{C}(1 \mathrm{~min} 30 \mathrm{sec})$. PCR products were directly sequenced on both strands with a CEQ2000 DNA analysis system using the CEQ-DTCS QuickStart kit (Beckman Coulter) The sequencing primers were the following: APOA5sF1 (5'-CCTTCGTCTCCTTCTTCCCCTAACC-3'), APOA5sR1 (5'-TGTGGAGAGGGACTAGGTAATCAGG-3'), APOA5sF2 (5'TGGCTCTTCTTTCAGGTGGGTCTCC-3'), APOA5sR2 (5'-CCAGCAGCGGCCACAGAGGTTGAG-3'), APOA5sF3 (5'-TGGGGACAAAGGAGATGAT-3'), APOA5-seqR3 (5'-GCCTTCACCTCCTCCAACTC-3'), APOA5sF4 (5'-GCAGCTGCAGGAGGAGTTGG-3'), APOA5sR4 (5'-AACTGGGCCTTGGTGTC), APOA5sF5 (5'-GGGGGAAGACACCAA-3'), APOA5sR5 (5'-TGCGGAGCCACACTG-3'), APOA5sF6 (5'-TCCACCCATACGCCGAGAGC-3'), APOA5sR6 (5'-GCGGAAAGCCTGAAGTCG-3'), APOA5sF7 (5'-CCCGGACCCCCAGATGCT-3'), APOA5sR7 (5'-AGGCTGTGAGTGATGTCTT-3'), APOA5sF8 (5'-AGTGGCAAGGTTCTGAG-3'), and APOA5sR8 (5'-AGACAGCAGCCCCTTTGGTG-3').

PCR-RFLP screening of the APOA5-Q139X mutation. Genomic DNA was PCR amplified with $0.1 \mu \mathrm{M} / 1$ of primers AV139XF (5'-TGCAGGAGGAGTTGGAGGAGGTGA-3') and AV139XR ( $5^{\prime}$-TGCACGCGCAGGGCCAG- $\left.3^{\prime}\right)$ as indicated earlier, except for an annealing temperature of $60^{\circ} \mathrm{C}$ and without DMSO. PCR products were subsequently digested with Pvu-II restriction enzyme (Roche Diagnostics Corp.) before gel electrophoresis analysis.

APOA5 haplotype analysis. To unambiguously assess haplotypes, $7040 \mathrm{bp}$ $A P O A 5$ PCR products were generated and subcloned in the PGEM-T vector system (Promega) prior to sequencing. The PCR primers were APOA5F9 (5'-GAGCTCAGGCCCATTCAAAACAA-3') and APOA5R9 (5'-GCTCACCAGGCTCTCGGCGTATG-3'). PCRs (35 cycles) were performed using the expanded high-fidelity PCR system as described by the manufacturer (Roche Diagnostics Corp.). Q139X-positive and -negative clones were sequenced with the following primers: APOA5F9, APOA5F10 (5'-CTAACTGTATGGCCCCAATCTAAT-3'), APOA5F11 (5'-TCTGCTGATGACTCCCAAAACTC-3'), APOA5F12 (5'-AAGAAAAAGCCCCTGCACTCAAAG-3'), APOA5F13 (5'-GATTGATTCAAGATGCATTTAGGAC-3') and APOA5R13 (5'-CCCCAGGAACTGGAGCGAAAGT-3'), APOA5sF1, APOA5sR1, APOA5sF2, and APOA5sR2. 


\section{Circulating apoB kinetic study}

Experimental protocol. A kinetic study of APOB 100 was performed in the 3 patients with the APOA5 mutation (AII1, AIII2, and BIII3). The results were compared with those of control normolipidemic men and women. The kinetic study was performed in fed state. Food intake, with a leucine-poor diet ( $1700 \mathrm{kcal} / \mathrm{d}$; $55 \%$ carbohydrates, $39 \%$ fats, and $7 \%$ proteins), was fractionated in small equal portions that were provided every 2 hours, starting 6 hours prior to the tracer infusion up to the end of the study, in order to avoid important variations in apolipoprotein plasma concentration as previously performed by other groups $(33,34)$. To determine the kinetic of APOB100, the subjects received an intravenous injection of a $0.7 \mathrm{mg} / \mathrm{kg}$ bolus of L- $\left[1-{ }^{13} \mathrm{C}\right]$ leucine $\left(99 \%{ }^{13} \mathrm{C}\right.$; Eurisotop) immediately followed by a 16 -hour constant infusion at $0.7 \mathrm{mg} / \mathrm{kg} / \mathrm{h}$. Blood samples were collected at hours 0 , $0.25,0.75,1,1.5,2,3,4,6,8,10,12,14,15$, and 16 . Serum was separated by centrifugation and stored at $4{ }^{\circ} \mathrm{C}$. Inhibitors of protease (aprotinin, $17 \mathrm{mg} / \mathrm{l}$ ) and bacterial growth (sodium azide, $500 \mathrm{mg} / \mathrm{l}$ ) were added to each sample.

Analytical procedures. Analytical procedures were performed as previously described in detail $(22,23,35)$.

Isolation of apolipoproteins. VLDL (density $[d]<1.006 \mathrm{~g} / \mathrm{ml})$, IDL $(1.006<d<$ $1.019)$ and $\operatorname{LDL}(1.019<d<1.063)$ were isolated from plasma by sequential flotation ultracentrifugation, using a 50.4 rotor in an L7 apparatus (Beckman Coulter). IDL and LDL fractions were then dialyzed against a $10 \mathrm{mM} / 1$ ammonium bicarbonate buffer $\mathrm{pH} 8.2$ containing $0.01 \%$ EDTA and $0.013 \%$ sodium azide. VLDL, IDL, and LDL fractions were delipidated with diethylether-ethanol, and APOB100 from each lipoprotein fraction was isolated by preparative SDS-PAGE (3\%). After staining with Coomassie blue R-250, APOB100 bands were excised from the polyacrylamide gels and hydrolyzed in $6 \mathrm{M} \mathrm{HCl}$ at $110^{\circ} \mathrm{C}$ for 16 hours under nitrogen vacuum. Samples were then lyophilized in a SpeedVac (Savant Instruments). Lyophilized samples were dissolved in 50\% acetic acid and applied to an AG-50W-X8 200-400 mesh cation exchange resin (Bio-Rad Laboratories); aa were recovered by elution with $4 \mathrm{~N} \mathrm{NH}_{4} \mathrm{OH}(34)$.

Determination of leucine enrichment by gas chromatograph/combustion/isotope ratio mass spectrometry (GC/C/IRMS). aa were converted to N-acetyl O-propyl (NAP) aa esters and analyzed with a Finnigan Mat Delta C isotope ratio mass spectrometer (Finnigan Mat) coupled to an HP 5890 series II gas chromatograph (Hewlett-Packard) (36), as previously described (37).

Modeling. Apolipoprotein kinetics data were expressed as tracer-to-trace mass ratios $(37,38) z(t)$, calculated as follows: $z(t)=e(t) / e_{i}-e(t)$, where $e_{i}$ is the tracer enrichment, $e(t)=a(t)-a N$, and $a(t)$ and $a N$ are the isotope abundance of the labeled and the unlabeled species, respectively.

The data were analyzed with the Simulation, Analysis, and Modeling (SAAM) II program (SAAM Institute Inc.) using a multicompartmental model (39). In the model chosen, VLDL apoB kinetic data are represented by compartments 1 and 2, plasma IDL apoB kinetic data by compartments 11 and 12, and LDL apoB kinetic data by compartment 21 . As the experiment was performed in the steady state, fractional synthetic rate equaled FCR (38).

Direct FCR of VLDL apoB and FCR from VLDL to IDL or LDL, expressed in pool/d, were calculated as follows: direct $\mathrm{FCR}_{\mathrm{VLDL}}=M_{2} k(0,2) /\left(M_{1}+M_{2}\right)$; $\mathrm{FCR}_{V L D L} \rightarrow \mathrm{IDL} \rightarrow \mathrm{LDL}=M_{1}[k(11,1)+k(21,1)] /\left(M_{1}+M_{2}\right)$, where $k(i, j)$ is the fractional transfer coefficient from compartment $j$ to $i$, and $M j$ represents the apoB mass (expressed as concentration/l plasma) of compartment $j$. Total
apoB VLDL FCR is the sum of direct FCR FLDL $_{V}$ and FCR FLDL $\rightarrow$ IDL $\rightarrow$ LDL $_{\text {(transfer }}$ to IDL/LDL). Direct FCR of IDL apoB and FCR from IDL to LDL were calculated as follows: direct $\mathrm{FCR}_{\mathrm{IDL}}=M_{12} k(0,12) /\left(M_{11}+M_{12}\right)$ and $\mathrm{FCR}_{\mathrm{IDL} \rightarrow \mathrm{LDL}}$ $=M_{11} k(21,11) /\left(M_{11}+M_{12}\right)$. Total apoB IDL FCR is the sum of direct FCR $\mathrm{FDL}_{\mathrm{IDL}}$

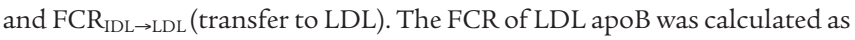
follows: $\mathrm{FCR}_{\mathrm{LDL}}=k(0,21)$. PRs of the APOB100 in each lipoprotein fraction were calculated as follows: $\mathrm{Pr}=\mathrm{apoB} F C R$ (for each lipoprotein fraction) $\times$ apoB pool size/body wt, where apoB pool size is calculated by multiplying the apoB concentration in the lipoprotein fraction (VLDL, IDL, or LDL) by the estimated plasma volume ( $4.5 \%$ of body wt).

LPL activity and Western blotting. Postheparin plasma was obtained 10 minutes after intravenous injection of heparin $(50 \mathrm{IU} / \mathrm{kg})$ and assay of lipase activity using a radio-labeled $14 \mathrm{C}$-triolein emulsion as previously described $(40,41)$. Human heat-inactivated serum $(10 \%)$ was used as a source of apoCII. To measure LPL activity, hepatic lipase was inhibited by preincubation with a specific goat polyclonal anti-serum (a gift from S. Griglio, U551 INSERM, Paris, France). LPL Western blotting was performed in postheparin plasma as previously described (40). In brief, postheparin plasma $(200 \mu \mathrm{l})$ was mixed with heparin-sepharose CL-6B (Pharmacia), incubated for 1 hour at $4^{\circ} \mathrm{C}$ in $0.1 \mathrm{M} / 1$ phosphate buffer, $\mathrm{pH} 7.2$, containing $0.15 \mathrm{M} / 1 \mathrm{NaCl}, 1 \mathrm{mM} / 1$ EDTA, $10 \%$ glycerol, and $10 \%$ diethyl p-nitrophenylphosphate. The slurry was washed twice prior to elution with 0.8 and $1.3 \mathrm{M} / 1 \mathrm{NaCl}$ buffer. Eluted fractions were TCA precipitated, and the pellet was analyzed on $10 \%$ SDS-PAGE electrophoresis and transferred to nitrocellulose. Blot was exposed to anti-LPL 5D2 mAb (1:500) and revealed with ECL (Amersham Biosciences).

Plasma Apoa5 assay and Western blot analysis. Apoa5 plasma concentrations were measured as previously described (25). For Western blot analysis, proteins were extracted from ultracentrifugation fractions as indicated in the kinetic study (22). Equal amounts of total proteins (100 $\mu \mathrm{g})$ were subjected to a $15 \%$ SDS-PAGE and then transferred onto a nitrocellulose membrane. The blots were first incubated with a goat anti-human Apoa5 (1:300) directed to the $\mathrm{N}$ terminal region, then with an anti-goat $\mathrm{IgG}$ horseradish peroxidase conjugate (1:1000; Sigma-Aldrich). Detection was performed by a chemiluminescent method (ECL, Amersham Biosciences). Normal control plasma was analyzed from normolipidemic subjects $(n=10)$.

\section{Acknowledgments}

We thank E. Rubin, F. Berthezene, G. Ponsin, and M. Lagarde for helpful discussion; D. Gaillard, V. Kerlan, O. Ziegler, N. Schneider, C. Clukas-Croisier, and G. Sautot for medical cooperation; M. Delay, E. Florentin, and C. Jacob for technical expertise; and J.D. Brunzell for the generous gift of 5D2 mAb. This work was supported by Hospices Civils de Lyon (HCL) and INSERM.

Received for publication January 14, 2005, and accepted in revised form July 12, 2005.

Address correspondence to: Christophe Marçais, Centre Hospitalier Lyon-Sud, Laboratoire de Biochimie, Batiment 3B, Niveau 1, Chemin du Grand Revoyet, 69495 Pierre-Bénite Cedex, France. Phone: 33-4-78-86-19-68; Fax: 33-4-78-86-66-54; E-mail: christophe.marcais@chu-lyon.fr.
1. Hokanson, J.E., and Austin M.A. 1996. Plasma
triglyceride level is a risk factor for cardiovascu-
lar disease independent of high-density lipopro-
tein cholesterol level: a meta-analysis of popula-
tion-based prospective studies. J. Cardiovasc. Risk.
3:213-219.
2. Chait, A., and Brunzell, J.D. 1990. Acquired hyperli-
pidemia (secondary dyslipoproteinemias). Endocri- nol. Metab. Clin. North Am. 19:259-278

3. Ma, Y., et al. 1994. High frequency of mutations in the human lipoprotein lipase gene in pregnancy-induced chylomicronemia: possible association with apolipoprotein E2 isoform. J. Lipid Res. 35:1066-1075

4. Keilson, L.M., Vary, C.P., Sprecher, D.L., and Renfrew, R. 1996. Hyperlipidemia and pancreatitis during pregnancy in two sisters with a mutation in the lipoprotein lipase gene. Ann. Intern. Med. 124:425-428

5. Marcais, C., et al. 2000. Severe hypertriglyceridaemia in type II diabetes: involvement of apoC-III Sst-I polymorphism, LPL mutations and apo E3 deficiency. Diabetologia. 43:1346-1352.

6. Pennacchio, L.A., et al. 2001. An apolipoprotein 
influencing triglycerides in humans and mice revealed by comp/arative sequencing. Science. 294:169-173.

7. van der Vliet, H.N., et al. 2001. Apolipoprotein A-V: a novel apolipoprotein associated with an early phase of liver regeneration. J. Biol. Chem. 276:44512-44520.

8. van der Vliet, H.N., et al. 2002. Adenoviral overexpression of apolipoprotein $\mathrm{A}-\mathrm{V}$ reduces serum levels of triglycerides and cholesterol in mice. Biochem. Biophys. Res. Commun. 295:1156-1159.

9. Pennacchio, L.A., et al. 2002. Two independent apolipoprotein A5 haplotypes influence human plasma triglyceride levels. Hum. Mol. Genet. 11:3031-3038.

10. Talmud, P.J., et al. 2002. Relative contribution of variation within the APOC3/A4/A5 gene cluster in determining plasma triglycerides. Hum. Mol. Genet. 11:3039-3046.

11. Olivier, M., et al. 2004. Haplotype analysis of the apolipoprotein gene cluster on human chromosome 11. Genomics. 83:912-923.

12. Oliva, C.P., et al. 2005. Inherited apolipoprotein A$\mathrm{V}$ deficiency in severe hypertriglyceridemia. Arterioscler. Thromb. Vasc. Biol. 25:411-417.

13. Baum, L., Tomlinson, B., and Thomas, G.N. 2003. APOA5-1131T $>$ C polymorphism is associated with triglyceride levels in Chinese men. Clin. Genet. 63:377-379.

14. Nabika, T., Nasreen, S., Kobayashi, S., and Masuda, J. 2002. The genetic effect of the apoprotein AV gene on the serum triglyceride level in Japanese. Atherosclerosis. 165:201-204.

15. Endo, K., et al. 2002. Association found between the promoter region polymorphism in the apolipoprotein $\mathrm{A}-\mathrm{V}$ gene and the serum triglyceride level in Japanese school children. Hum. Genet. 111:570-572.

16. Aouizerat, B.E., et al. 2003. Genetic analysis of a polymorphism in the human apoA-V gene: effect on plasma lipids. J. Lipid Res. 44:1167-1173.

17. Ward, K.J., et al. 2003. Genetic variants in apolipoprotein $\mathrm{AV}$ alter triglyceride concentrations in pregnancy. Lipids Health Dis. 2:2-9.

18. Ribalta, J., et al. 2002. Newly identified apolipoprotein $\mathrm{AV}$ gene predisposes to high plasma triglycerides in familial combined hyperlipidemia. Clin. Chem. 48:1597-1600.

19. Eichenbaum-Voline, S., et al. 2004. Linkage and association between distinct variants of the
APOA1/C3/A4/A5 gene cluster and familial combined hyperlipidemia. Arterioscler. Thromb. Vasc. Biol. 24:167-174.

20. Horinek, A., et al. 2003. T-1131>C polymorphism within the apolipoprotein AV gene in hypertriglyceridemic individuals. Atherosclerosis. 167:369-370

21. Schaefer, J.R., et al. 2004. Hyperlipidemia in patients with apolipoprotein E $2 / 2$ phenotype: apolipoprotein A5 S19W mutation as a cofactor. Clin. Chem. 50:2214.

22. Duvillard, L., et al. 2000. Metabolic abnormalities of apolipoprotein B-containing lipoprotein in non insulin dependent diabetes: a stable isotope study. Eur. J. Clin. Invest. 30:685-694

23. Duvillard, L., Pont, F., Florentin, E., Gambert, P., and Vergès, B. 2000. Significant improvement of apolipoprotein B-containing lipoprotein metabolism by insulin treatment in patients with noninsulin-dependent diabetes mellitus. Diabetologia. 43:27-35.

24. Merkel, M., et al. 2005. Apolipoprotein AV accelerates plasma hydrolysis of triglyceride-rich lipoproteins by interaction with proteoglycan bound lipoprotein lipase. J. Biol. Chem. 280:21553-21560

25. Fruchart-Najib, J., et al. 2004. Mechanism of triglyceride lowering in mice expressing human apolipoprotein A5. Biochem. Biophys. Res. Commun. 319:397-404

26. Schaap, F.G., et al. 2004. ApoAV reduces plasma triglycerides by inhibiting very low density lipoprotein-triglyceride (VLDL-TG) production and stimulating lipoprotein lipase-mediated VLDL-TG hydrolysis. J. Biol. Chem. 279:27941-27947.

27. Weinberg, R.B., et al. 2003. Structure and interfacial properties of human apolipoprotein A-V. J. Biol. Chem. 278:34438-34444.

28. Lookene, A. Beckstead, J.A., Nilsson, S., Olivecrona, G., and Ryan, R.O. 2005. Apolipoprotein A-V-heparin interactions: implications for plasma lipoprotein metabolism. J. Biol. Chem. 280:25383-25387.

29. Taskinen, M.R., Nikkila, E.A., Kuusi, T., and Harmo, K. 1982. Lipoprotein lipase activity and serum lipoproteins in untreated type 2 (insulinindependent) diabetes associated with obesity. Diabetologia. 22:46-50.

30. Eriksson, J.W., Buren, J., Svensson, M., Olivecrona, T., and Olivecrona, G. 2003. Postprandial regulation of blood lipids and adipose tissue lipoprotein lipase in type 2 diabetes patients and healthy control subjects. Atherosclerosis. 166:359-367
31. Pruneta-Deloche, V., et al. 2004. Alteration in lipoprotein lipase activity bound to triglyceriderich lipoproteins in the postprandial state in type 2 diabetes. J. Lipid Res. 45:859-865.

32. Brunzell, J.D. 1995. Familial lipoprotein lipase deficiency and other causes of the chylomicronemia syndrome. In The metabolic and molecular bases of inherited disease. Volume 2. C.R. Scriver, A.L. Beaudet, W.S. Sly, and D. Valle, editors. McGraw-Hill. New York, New York, USA. 1913-1932.

33. Taskinen, M.R., Packard, C.J., and Sheperd, J. 1990. Effect of insulin therapy on metabolic fate of apolipoprotein B-containing lipoproteins in NIDDM. Diabetes. 39:1017-1027.

34. Vergès, B., et al. 1994. In vivo metabolism of apolipoprotein A-IV in severe hypertriglyceridemia: a combined radiotracer and stable isotope kinetic study. J. Lipid Res. 35:2280-2291.

35. Pont, F., Duvillard, L., Florentin, E., Gambert, P., and Vergès, B. 2002. Early kinetic abnormalities of apolipoprotein B-containing lipoproteins in insulin resistant women with abdominal obesity. Arterioscler. Thromb. Vasc. Biol. 22:1726-1732.

36. Yarasheski, K.E., Smith, K., Rennie, M.J., and Bier, D.M. 1992. Measurement of muscle protein fractional synthetic rate by capillary gas chromatography/combustion isotope ratio mass spectrometry. Biol. Mass Spectrom. 21:486-490.

37. Pont, F., et al. 1997. Isotope ratio mass spectrometry, compared with conventional mass spectrometry in kinetic studies at low and high enrichment levels: application to lipoprotein kinetics. Anal. Biochem. 248:277-287.

38. Cobelli, C., Toffolo, G., and Foster, D.M. 1992. Tracer-to-tracee ratio for analysis of stable isotope tracer data: link with radioactive kinetic formalism. Am. J. Physiol. 262:E968-E975.

39. Barrett, P.H.R., et al. 1998. SAAM II: simulation, analysis and modeling software for tracer and pharmacokinetic studies. Metabolism. 47:484-492.

40. Pruneta, V., et al. 1997. Characterization of a new case of autoimmune type I hyperlipidemia: longterm remission under immunosuppressive therapy. J. Clin. Endocrinol. Metab. 82:791-796.

41. Pruneta, V., et al. 2001. Ex vivo measurement of lipoprotein lipase-dependent very low density lipoprotein (VLDL)-triglyceride hydrolysis in human VLDL: an alternative to the postheparin assay of lipoprotein lipase activity? J. Clin. Endocrinol. Metab. 86:797-803. 